\title{
Staggered-Grid Finite Difference Method with Variable-Order Accuracy for Porous Media
}

\author{
Jinghuai Gao ${ }^{1,2}$ and Yijie Zhang ${ }^{1,2}$ \\ ${ }^{1}$ Institute of Wave and Information, Xi'an Jiaotong University, Xi'an 710049, China \\ ${ }^{2}$ National Engineering Laboratory for Offshore Oil Exploration, Xian 710049, China \\ Correspondence should be addressed to Jinghuai Gao; jhgao@mail.xjtu.edu.cn
}

Received 9 January 2013; Accepted 7 April 2013

Academic Editor: Alex Elias-Zuniga

Copyright (c) 2013 J. Gao and Y. Zhang. This is an open access article distributed under the Creative Commons Attribution License, which permits unrestricted use, distribution, and reproduction in any medium, provided the original work is properly cited.

\begin{abstract}
The numerical modeling of wave field in porous media generally requires more computation time than that of acoustic or elastic media. Usually used finite difference methods adopt finite difference operators with fixed-order accuracy to calculate space derivatives for a heterogeneous medium. A finite difference scheme with variable-order accuracy for acoustic wave equation has been proposed to reduce the computation time. In this paper, we develop this scheme for wave equations in porous media based on dispersion relation with high-order staggered-grid finite difference (SFD) method. High-order finite difference operators are adopted for low-velocity regions, and low-order finite difference operators are adopted for high-velocity regions. Dispersion analysis and modeling results demonstrate that the proposed SFD method can decrease computational costs without reducing accuracy.
\end{abstract}

\section{Introduction}

Seismic wave modeling for porous media is usually used to study the properties of rocks and to characterize the seismic response of geologic formation. Furthermore, the theory about poroelasticity is a suitable characterization for the environment of hydrocarbon reservoirs.

The most popular theory about poroelasticity is developed by Biot [1-3], which is the basis for solving the wave propagation problems in porous media. A variety of different numerical methods have been used for poroelasticity modeling [4, 5], such as spectral method [6], finite difference method [7], time domain method [8], discontinuous Galerkin method [9], and finite volume method [10]. Owing to the low memory requirement and computational cost, finite difference methods $[11,12]$ are widely applied for porous wave equations.

To improve the efficiency and accuracy, several variants of finite difference methods have been investigated to simulate porous media $[13,14]$. These include implicit finite difference method [15], variable-grids [16], irregular-grids, staggeredgrids [17], discontinuous-grids [18], quadrangle-grids [19], rotated-staggered-grids [20] finite difference methods, and spatially varying time steps finite difference methods [21]. In order to avoid the spurious reflection from the artificial boundaries in finite difference modeling, perfectly matched layer (PML) absorbing boundary conditions $[22,23]$ have been widely used.

Compared with the numerical modeling of acoustic or elastic wave propagation, the porous wave propagation may spend more computation time. As we know, the wavelength in a lower velocity medium is smaller than that in a highvelocity medium with a fixed source frequency [24] and the accuracy of high-velocity regions is higher than that of low-velocity regions with a fixed-order finite difference operator for heterogeneous media. In addition, high-order finite difference operators provide better accuracy than loworder finite difference operators. Therefore, we can adopt high-order finite difference operators for low-velocity regions and low-order finite difference operators for high-velocity regions to reduce the computational costs.

The remainder of this paper is organized as follows. In Section 2, Biot's equations are reformulated into the firstorder, velocity-stress system; we derive the dispersion relation based on high-order staggered-grid finite difference method for porous media. In Section 3, we propose a method to 
determine the orders of accuracy for different velocities automatically. The validity of this method is demonstrated by dispersion analysis. In Section 4 , we use the staggeredgrid finite difference method with fixed- and variable-order accuracy to simulate porous media. The modeling results demonstrate the efficiency of our method. We state the conclusion of this paper in Section 5.

\section{Dispersion Relation for Porous Media}

Biot established the dynamic equations in a porous elastic solid saturated by a compressible viscous fluid, and predicted that two dilatational waves and one rotational wave exist in the fluid-saturated porous solid. A first-order hyperbolic system [25-27] is developed, which is equivalent to Biot's equations, whose vector of unknowns consists of the solid and fluid particle velocity components, the solid stress components, and the fluid pressure. According to Biot's theory, the equations of motion for $2 \mathrm{D}$ porous media are given by

$$
\begin{aligned}
& \frac{\partial v_{x}}{\partial t}=R_{22}\left(\frac{\partial \tau_{x x}}{\partial x}+\frac{\partial \tau_{x z}}{\partial z}\right)-R_{12} \frac{\partial s}{\partial x}+B_{2}\left(w_{x}-v_{x}\right), \\
& \frac{\partial v_{z}}{\partial t}=R_{22}\left(\frac{\partial \tau_{x z}}{\partial x}+\frac{\partial \tau_{z z}}{\partial z}\right)-R_{12} \frac{\partial s}{\partial z}+B_{2}\left(w_{z}-v_{z}\right), \\
& \frac{\partial w_{x}}{\partial t}=R_{11} \frac{\partial s}{\partial x}-R_{12}\left(\frac{\partial \tau_{x x}}{\partial x}+\frac{\partial \tau_{x z}}{\partial z}\right)-B_{1}\left(w_{x}-v_{x}\right), \\
& \frac{\partial w_{z}}{\partial t}=R_{11} \frac{\partial s}{\partial z}-R_{12}\left(\frac{\partial \tau_{x z}}{\partial x}+\frac{\partial \tau_{z z}}{\partial z}\right)-B_{1}\left(w_{z}-v_{z}\right), \\
& \frac{\partial \tau_{x x}}{\partial t}=P \frac{\partial v_{x}}{\partial x}+(P-2 N) \frac{\partial v_{z}}{\partial z}+Q\left(\frac{\partial w_{x}}{\partial x}+\frac{\partial w_{z}}{\partial z}\right) \\
& +q_{x}\left(w_{x}-v_{x}\right)+q_{z}\left(w_{z}-v_{z}\right) \\
& \frac{\partial \tau_{z z}}{\partial t}=(P-2 N) \frac{\partial v_{x}}{\partial x}+P \frac{\partial v_{z}}{\partial z}+Q\left(\frac{\partial w_{x}}{\partial x}+\frac{\partial w_{z}}{\partial z}\right) \\
& +q_{x}\left(w_{x}-v_{x}\right)+q_{z}\left(w_{z}-v_{z}\right) \\
& \frac{\partial \tau_{x z}}{\partial t}=N\left(\frac{\partial v_{x}}{\partial z}+\frac{\partial v_{z}}{\partial x}\right) \\
& \frac{\partial s}{\partial t}=Q\left(\frac{\partial v_{x}}{\partial x}+\frac{\partial v_{z}}{\partial z}\right)+R\left(\frac{\partial w_{x}}{\partial x}+\frac{\partial w_{z}}{\partial z}\right)+r_{x}\left(w_{x}-v_{x}\right) \\
& +r_{z}\left(w_{z}-v_{z}\right) \text {, }
\end{aligned}
$$

where $\left(v_{x}, v_{z}\right)$ is the solid particle velocity vector, $\left(w_{x}, w_{z}\right)$ is the fluid particle velocity vector, and $\left(\tau_{x x}, \tau_{x z}, \tau_{z z}\right)$ is the solid stress tensor. $s$ is related to the fluid pressure $p$ and the porosity $\phi$ according to

$$
s=-\phi p
$$

The coefficients $P, Q, R$, and $N$ are Biot's elastic constants. $P$ and $N$ correspond to the familiar Lame coefficients in the theory of elasticity. The coefficient $R$ is a measure of the pressure required on the fluid to force a certain volume of the fluid into the aggregate, while the total volume remains constant. The coefficient $Q$ is the nature of a coupling between the volume change of solid and that of the fluid. And $\rho_{11}$, $\rho_{12}$, and $\rho_{22}$ are mass coefficients which take into account the fact that the relative fluid flow through the pores is not uniform.

The coefficient $b$ is related to Darcy's coefficient of permeability $\kappa$ by

$$
b=\frac{\mu \phi^{2}}{\kappa},
$$

where $\mu$ is the fluid viscosity.

The other parameters in (1)-(8) are defined as $R_{i j}=$ $\rho_{i j} /\left(\rho_{11} \rho_{22}-\rho_{12}^{2}\right), B_{1}=b\left(R_{11}+R_{12}\right), B_{2}=b\left(R_{12}+R_{22}\right)$, $q_{x}=(Q / \phi)(\partial \phi / \partial x), q_{z}=(Q / \phi)(\partial \phi / \partial z), r_{x}=(R / \phi)(\partial \phi / \partial x)$, and $r_{z}=(R / \phi)(\partial \phi / \partial z)$.

In staggered-grid scheme, particle velocity components, solid stress components, and fluid pressure are located at different points, which may be discretized as $\left(v_{x}\right)_{p, q}^{n}$, $\left(w_{x}\right)_{p, q}^{n},\left(v_{z}\right)_{p+1 / 2, q+1 / 2}^{n},\left(w_{z}\right)_{p+1 / 2, q+1 / 2}^{n},\left(\tau_{x x}\right)_{p+1 / 2, q}^{n-1 / 2},\left(\tau_{z z}\right)_{p+1 / 2, q^{\prime}}^{n-1 / 2}$, $(s)_{p+1 / 2, q}^{n-1 / 2}$, and $\left(\tau_{x z}\right)_{p, q+1 / 2}^{n-1 / 2}$. The subscripts refer to the spatial indices, and the superscripts refer to the time indices. Thus, with a grid spacing $h$ and time step $\tau$, for example, the expression $\left(v_{x}\right)_{p, q}^{n}$ represents the $x$ component of the solid particle velocity evaluated at the point $[x+p h, z+q h]$ and at the time $t+n \tau$.

The Appendix gives the discretization of the derivatives in (1)-(8) based on high-order staggered-grid finite difference scheme. Using the plane wave theory, we let

$$
\begin{gathered}
\left(v_{x}\right)_{p, q}^{n}=A e^{i\left[k_{x}(x+p h)+k_{z}(z+q h)-\omega(t+n \tau)\right]}, \\
\left(w_{x}\right)_{p, q}^{n}=C e^{i\left[k_{x}(x+p h)+k_{z}(z+q h)-\omega(t+n \tau)\right]}, \\
\left(v_{z}\right)_{p+1 / 2, q+1 / 2}^{n}=D e^{i\left[k_{x}(x+(p+1 / 2) h)+k_{z}(z+(q+1 / 2) h)-\omega(t+n \tau)\right]}, \\
\left(w_{z}\right)_{p+1 / 2, q+1 / 2}^{n}=E e^{i\left[k_{x}(x+(p+1 / 2) h)+k_{z}(z+(q+1 / 2) h)-\omega(t+n \tau)\right]}, \\
\left(\tau_{x x}\right)_{p+1 / 2, q}^{n+1 / 2}=F e^{i\left[k_{x}(x+(p+1 / 2) h)+k_{z}(z+q h)-\omega(t+(n+1 / 2) \tau)\right]}, \\
\left(\tau_{z z}\right)_{p+1 / 2, q}^{n+1 / 2}=G e^{i\left[k_{x}(x+(p+1 / 2) h)+k_{z}(z+q h)-\omega(t+(n+1 / 2) \tau)\right]}, \\
(s)_{p+1 / 2, q}^{n+1 / 2}=H e^{i\left[k_{x}(x+(p+1 / 2) h)+k_{z}(z+q h)-\omega(t+(n+1 / 2) \tau)\right]}, \\
\left(\tau_{x z}\right)_{p, q+1 / 2}^{n+1 / 2}=K e^{i\left[k_{x}(x+p h)+k_{z}(z+(q+1 / 2) h)-\omega(t+(n+1 / 2) \tau)\right]}
\end{gathered}
$$

where $\omega$ is the angular frequency, $\left(k_{x}, k_{z}\right)$ is the wavenumber vector, and $i=\sqrt{-1}$. 
Substituting (A.1)-(A.20) of the Appendix and (11) into (1)-(8) and simplifying,

$$
\begin{gathered}
g A=R_{22}\left(f_{x} F+f_{z} K\right)-R_{12} f_{x} H, \\
g D=R_{22}\left(f_{x} K+f_{z} G\right)-R_{12} f_{z} H, \\
g C=R_{11} f_{x} H-R_{12}\left(f_{x} F+f_{z} K\right), \\
g E=R_{11} f_{z} H-R_{12}\left(f_{x} K+f_{z} G\right), \\
g F=P f_{x} A+Q\left(f_{x} C+f_{z} E\right)+(P-2 N) f_{z} D, \\
g G=(P-2 N) f_{x} A+Q\left(f_{x} C+f_{z} E\right)+P f_{z} D, \\
g K=N\left(f_{x} D+f_{z} A\right), \\
g H=Q\left(f_{x} A+f_{z} D\right)+R\left(f_{x} C+f_{z} E\right)
\end{gathered}
$$

$$
\begin{gathered}
g=-\sin \left(\frac{\omega \tau}{2}\right), \\
f_{x}=\frac{\tau}{h} \sum_{m=1}^{M} a_{m} \sin \left(\left(m-\frac{1}{2}\right) k_{x} h\right), \\
f_{z}=\frac{\tau}{h} \sum_{m=1}^{M} a_{m} \sin \left(\left(m-\frac{1}{2}\right) k_{z} h\right),
\end{gathered}
$$

where $a_{m}(m=1,2, \ldots, M)$ are the SFD coefficients [28] and $2 M$ corresponds to the order of accuracy.

Equations (12) can be rewritten into matrix form

$$
\left[\begin{array}{cccccccc}
0 & 0 & 0 & 0 & R_{22} f_{x} & 0 & R_{22} f_{z} & -R_{12} f_{x} \\
0 & 0 & 0 & 0 & 0 & R_{22} f_{z} & R_{22} f_{x} & -R_{12} f_{z} \\
0 & 0 & 0 & 0 & -R_{12} f_{x} & 0 & -R_{12} f_{z} & R_{11} f_{x} \\
0 & 0 & 0 & 0 & 0 & -R_{12} f_{z} & -R_{12} f_{x} & R_{11} f_{z} \\
P f_{x} & (P-2 N) f_{z} & Q f_{x} & Q f_{z} & 0 & 0 & 0 & 0 \\
(P-2 N) f_{x} & P f_{z} & Q f_{x} & Q f_{z} & 0 & 0 & 0 & 0 \\
N f_{z} & N f_{x} & 0 & 0 & 0 & 0 & 0 & 0 \\
Q f_{x} & Q f_{z} & R f_{x} & R f_{z} & 0 & 0 & 0 & 0
\end{array}\right]\left[\begin{array}{c}
A \\
D \\
C \\
E \\
F \\
G \\
K \\
H
\end{array}\right]=g\left[\begin{array}{c}
A \\
D \\
C \\
E \\
F \\
G \\
K \\
H
\end{array}\right] .
$$

That is, $\mathbf{W} \boldsymbol{\xi}=\boldsymbol{g} \boldsymbol{\xi}, \boldsymbol{g}$ is the eigenvalue of the matrix $\mathbf{W}$, we obtain

$$
\begin{gathered}
g^{2}=0, \\
g^{2}=R_{22} N\left(f_{x}^{2}+f_{z}^{2}\right), \\
g^{2}=\left(Z+\frac{\sqrt{W}}{2}\right)\left(f_{x}^{2}+f_{z}^{2}\right), \\
g^{2}=\left(Z-\frac{\sqrt{W}}{2}\right)\left(f_{x}^{2}+f_{z}^{2}\right),
\end{gathered}
$$

where $Z=(1 / 2) R_{11} R+(1 / 2) R_{22} P-R_{12} Q$ and $W=R^{2} R_{11}^{2}-$ $2 P R R_{11} R_{22}-4 R Q R_{11} R_{12}+P^{2} R_{22}^{2}-4 P Q R_{22} R_{12}+4 P R R_{12}^{2}+$ $4 Q^{2} R_{11} R_{22}$.

For porous media, Biot [1] derived the velocities of fast $\mathrm{P}$ wave, $S$ wave, and slow $P$ wave. The velocity of $S$ wave is

$$
v_{\mathrm{S}}=\left[\frac{N}{\rho_{11}\left(1-\rho_{12}^{2} /\left(\rho_{11} \rho_{22}\right)\right)}\right]^{1 / 2} .
$$

In order to derive the velocities of fast $\mathrm{P}$ wave and slow $\mathrm{P}$ wave, we introduce a reference velocity $v_{c}$ defined by

$$
v_{c}^{2}=\frac{H}{\rho} .
$$

The following parameters are introduced:

$$
\begin{array}{lll}
\sigma_{11}=\frac{P}{H}, & \sigma_{22}=\frac{R}{H}, & \sigma_{12}=\frac{Q}{H}, \\
\gamma_{11}=\frac{\rho_{11}}{\rho}, & \gamma_{22}=\frac{\rho_{22}}{\rho}, & \gamma_{12}=\frac{\rho_{12}}{\rho},
\end{array}
$$

where $H=P+R+2 Q$ and $\rho=\rho_{11}+2 \rho_{12}+\rho_{22}$.

For an equation of $z$

$$
\begin{aligned}
& \left(\sigma_{11} \sigma_{22}-\sigma_{12}^{2}\right) z^{2}-\left(\sigma_{11} \gamma_{22}+\sigma_{22} \gamma_{11}-2 \sigma_{12} \gamma_{12}\right) z \\
& +\left(\gamma_{11} \gamma_{22}-\gamma_{12}^{2}\right)=0
\end{aligned}
$$

there are two roots $z_{1}$ and $z_{2}$ corresponding to two velocities $v_{1}, v_{2}$,

$$
\begin{aligned}
& v_{1}^{2}=\frac{v_{c}^{2}}{z_{1}}, \\
& v_{2}^{2}=\frac{v_{c}^{2}}{z_{2}} .
\end{aligned}
$$

The larger velocity between $v_{1}$ and $v_{2}$ is the velocity of fast $\mathrm{P}$ wave $v_{\mathrm{P} \text { fast }}$, and the lower is the velocity of slow $\mathrm{P}$ wave $v_{\text {P_slow. }}$. 
Comparing (16)-(18) with (19), (23), and (24), (16)-(18) can be expressed as

$$
\begin{aligned}
& g^{2}=v_{\mathrm{S}}^{2}\left(f_{x}^{2}+f_{z}^{2}\right), \\
& g^{2}=v_{\mathrm{P}_{\text {slow }}}^{2}\left(f_{x}^{2}+f_{z}^{2}\right), \\
& g^{2}=v_{\mathrm{P}_{\text {fast }}}^{2}\left(f_{x}^{2}+f_{z}^{2}\right),
\end{aligned}
$$

where $v_{\mathrm{P}_{-} \text {fast }}, v_{\mathrm{P}_{-} \text {slow }}$, and $v_{\mathrm{S}}$ are the velocities of fast $\mathrm{P}$ wave, slow $\mathrm{P}$ wave, and S wave, respectively. Equations (25)-(27) are the dispersion relations based on high-order staggered-grid finite difference method for porous media.

\section{SFD Method with Variable-Order Accuracy}

The source frequency is usually fixed in the modeling so that the wavelength in a low-velocity region is smaller than that in a high-velocity region. For a heterogeneous model, the accuracy of high-velocity regions is higher than that of lowvelocity regions with a fixed-order finite difference operator to calculate the space derivatives. Moreover, high-order finite difference operator provides better accuracy than that of low-order finite difference operator. Therefore, we can adopt high-order finite difference operators for low-velocity regions and low-order finite difference operators for high-velocity regions. Note that three velocities exist in each grid for porous media, and we should choose the minimum velocity among them to determine the order of accuracy.

Substitute (13) into (25), (26), or (27), and let $k_{x}=k \cos \theta$ and $k_{z}=k \sin \theta$, where $\theta$ is a propagation direction angle of the plane wave. Dispersion relation of the minimum velocity can be written as

$$
\begin{aligned}
& \left(\sum_{m=1}^{M} a_{m} \sin ((m-0.5) k h \cos \theta)\right)^{2} \\
& \quad+\left(\sum_{m=1}^{M} a_{m} \sin ((m-0.5) k h \sin \theta)\right)^{2}=\left(r^{-1} \sin (0.5 \omega \tau)\right)^{2},
\end{aligned}
$$

where $r=v \tau / h$ and $v=\min \left(v_{\mathrm{S}}, v_{\mathrm{P}_{\text {_slow }}}, v_{\mathrm{P}_{\text {fast }}}\right)$.

Grid dispersion parameter in SFD modeling is defined by using (28),

$$
\delta_{\text {wave }}(\theta)=\frac{v_{\mathrm{FD}_{\text {wave }}}}{v_{\text {wave }}}=\frac{2}{r_{\text {wave }} k h} \sin ^{-1}\left(r_{\text {wave }} \sqrt{q}\right)
$$

with

$$
\begin{aligned}
q= & \left(\sum_{m=1}^{M} a_{m} \sin ((m-0.5) k h \cos \theta)\right)^{2} \\
& +\left(\sum_{m=1}^{M} a_{m} \sin ((m-0.5) k h \sin \theta)\right)^{2},
\end{aligned}
$$

where the subscript wave represents slow $\mathrm{P}$ wave, $\mathrm{S}$ wave, or fast $\mathrm{P}$ wave. If $\delta$ in (29) equals unity, there is no dispersion. If $\delta$ is larger or less than unity, the dispersion will occur.
Figure 1(a) shows the variation of the dispersion parameter $\delta$ with $k h$ for the same order of accuracy but different velocities. From this figure it can be noted that the dispersion curves are nearly consistent for different velocities. As the independent variable $k h$ is related to velocity, we can change $k h$ into $f$, which is independent of velocity. We plot the variation of the dispersion parameter $\delta$ with $f$ in Figure 1(b). It can be concluded that the numerical dispersion for highfrequency components reduces with the increase of velocity for fixed $M$ and $h$. As $k h$ ranges from 0 to $\pi$ (at Nyquist frequency), $f$ changes from 0 to $v / 2 h$. For different velocities, the values of maximum frequency are different. Note that the source frequency in the modeling is predetermined and is usually correlated with the minimum velocity for fixed-grid size. Therefore, we can reduce the order of accuracy for the high-velocity regions and achieve nearly the same accuracy as the high-order finite difference operators for the low-velocity regions.

The difference between SFD propagation time and exact propagation time through one grid could describe the error due to the numerical dispersion in SFD scheme,

$$
\varepsilon=\frac{h}{v_{\mathrm{FD}}}-\frac{h}{v}=\frac{h}{v}\left(\delta^{-1}-1\right) .
$$

If $\varepsilon$ equals zero, there is no dispersion; when $\varepsilon$ is much greater or less than zero, large dispersion will occur. Following the definition of SFD error, $\varepsilon$ is related to $v, M$, and $f$. Figure 1(c) illustrates that the SFD error for low velocity is larger than that for high velocity with a certain frequency, which implies that we can reduce the order of accuracy for the high-velocity regions without reducing the accuracy.

Here, we adopt a method [24] to determine the orders of accuracy for different velocities adaptively.

For the given maximum frequency $f_{\max }$ and the maximum error $\eta$, the following inequality is satisfied:

$$
|\varepsilon(v, M, f)| \leq \eta, \quad \text { when } f \leq f_{\max } .
$$

The maximum frequency $f_{\max }$ is related to the source frequency, and the maximum error $\eta$ should be larger than the maximum value of $\varepsilon$.

Figure 2 shows dispersion curves for different orders of accuracy and different velocities; the involved parameters are $v=500,1500,2500,3500,4500 \mathrm{~m} / \mathrm{s}, \tau=0.0001 \mathrm{~s}, h=2 \mathrm{~m}$, $f_{\max }=30 \mathrm{~Hz}$, and $\eta=10^{-9}$. The values of $M$ determined by (32) are $6,3,3,2$, and 2 for the five different velocities, respectively. From this figure it can be noted that the loworder finite difference operators can be adopted for highvelocity regions to obtain an accuracy that is better than or equal to the accuracy of the high-order finite difference operators for low-velocity regions.

The orders of accuracy with different velocities and maximum errors are presented in Figure 3. The figure demonstrates that the orders of accuracy generally decrease with the increase of velocities. In addition, the orders of accuracy determined by (32) are dependent on the time step $\tau$, the grid spacing $h$, and the maximum frequency $f_{\max }$. 


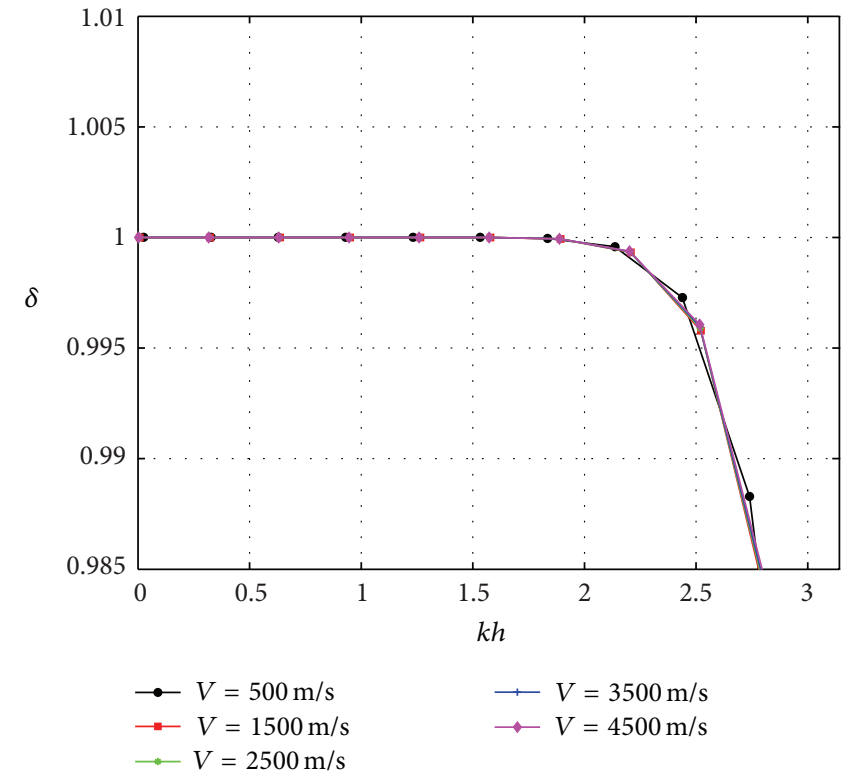

(a)

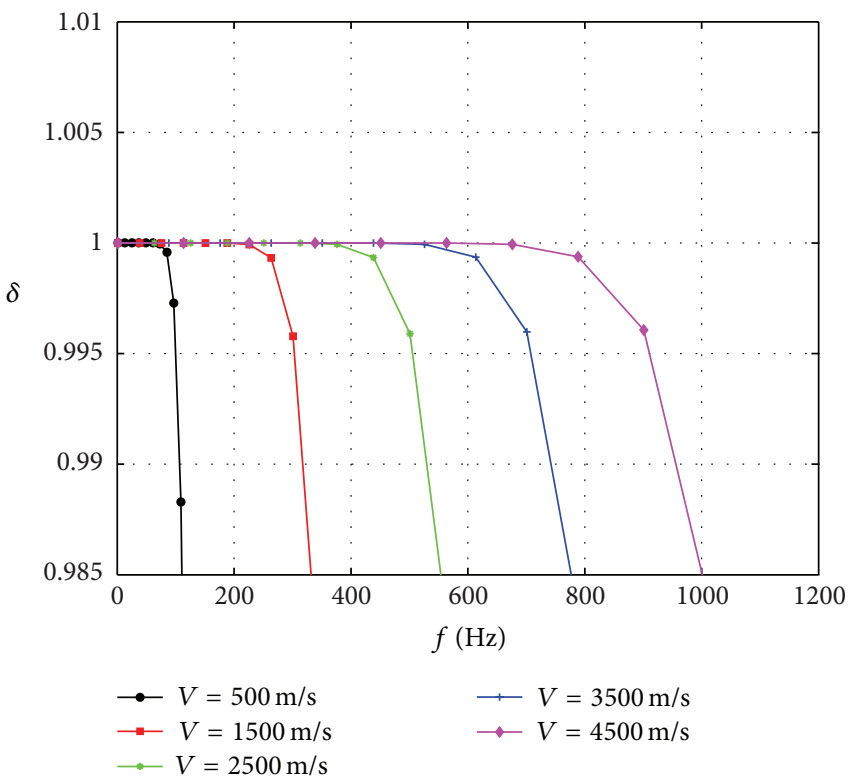

(b)

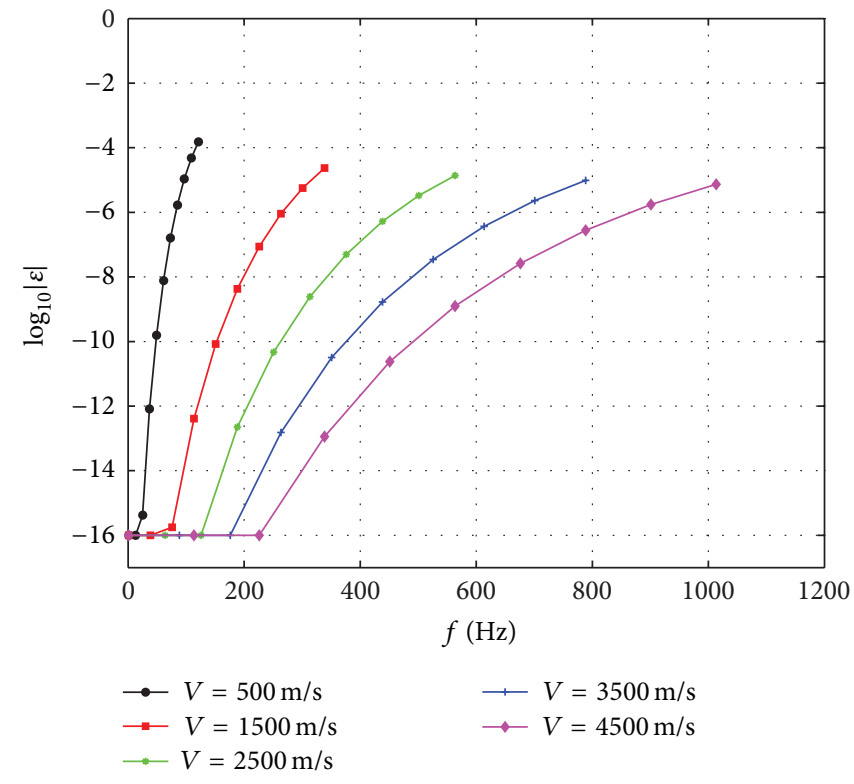

(c)

Figure 1: Dispersion curves for the same order of accuracy but different velocities. (a) Variation of dispersion parameter $\delta$ with $k h$, (b) variation of dispersion parameter $\delta$ with $f$, (c) variation of dispersion parameter $\log _{10}|\varepsilon|$ with $f$. Here, $v=500,1500,2500,3500,4500 \mathrm{~m} / \mathrm{s}$, $\tau=0.0001 \mathrm{~s}, h=2 \mathrm{~m}$, and $M=10$. When $\log _{10}|\varepsilon|<-16$, we set $\log _{10}|\varepsilon|=-16$.

TABLE 1: Parameters for the horizontally layered porous model.

\begin{tabular}{|c|c|c|c|c|c|c|c|c|c|c|c|c|}
\hline & $\rho_{12}$ & $\begin{array}{c}\rho_{11} \\
\left(\mathrm{~kg} / \mathrm{m}^{3}\right) \\
\end{array}$ & $\rho_{22}$ & $P$ & \multicolumn{3}{|c|}{$\left(\mathrm{N} / \mathrm{m}^{2}\right)$} & $\begin{array}{c}b \\
\left(\mathrm{Nsm}^{4}\right)\end{array}$ & $\phi$ & $v_{\text {P_fast }}$ & $\begin{array}{c}v_{S} \\
(\mathrm{~m} / \mathrm{s})\end{array}$ & $\overline{v_{\text {P_slow }}}$ \\
\hline 1 & -260 & 1947 & 520 & $5.3 e 9$ & $5.2 e 8$ & $3.8 e 8$ & $1.8 e 9$ & $1 e 3$ & 0.25 & 1856 & 1005 & 733 \\
\hline 2 & -104 & 2174 & 208 & $7.6 e 9$ & $2.4 e 8$ & $1.4 e 8$ & $2.6 e 9$ & $1 e 3$ & 0.1 & 1950 & 1100 & 764 \\
\hline 3 & -104 & 2384 & 156 & $9.2 e 9$ & $2.1 e 8$ & $3.4 e 7$ & $3.1 e 9$ & $1 e 3$ & 0.05 & 2030 & 1162 & 514 \\
\hline 4 & -31 & 2446 & 62 & $1.2 e 10$ & $1.1 e 8$ & $3.9 e 7$ & $4.1 e 9$ & $1 e 3$ & 0.03 & 2276 & 780 & 1298 \\
\hline 5 & -330 & 2092 & 660 & $7.8 e 9$ & $9.5 e 8$ & $5.3 e 8$ & $2.9 e 9$ & $1 e 3$ & 0.33 & 2215 & 1232 & 718 \\
\hline 6 & -100 & 2430 & 255 & $5.5 e 10$ & $7 e 8$ & $7.9 e 8$ & $1.2 e 10$ & $7 e 5$ & 0.25 & 4860 & 2240 & 1740 \\
\hline
\end{tabular}




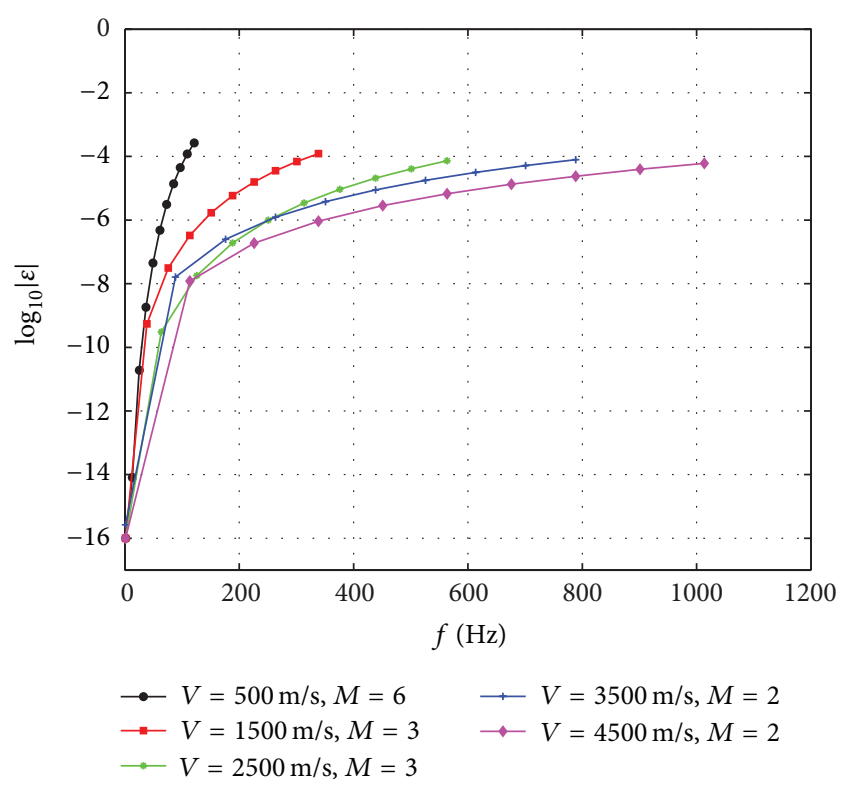

FIGURE 2: Dispersion curves for different orders of accuracy and velocities. Here, $v=500,1500,2500,3500,4500 \mathrm{~m} / \mathrm{s}, \tau=0.0001 \mathrm{~s}$, $h=2 \mathrm{~m}, f_{\max }=30 \mathrm{~Hz}, \eta=10^{-9}$, and $M=6,3,3,2$, and 2 determined by (32). When $\log _{10}|\varepsilon|<-16$, we set $\log _{10}|\varepsilon|=-16$.

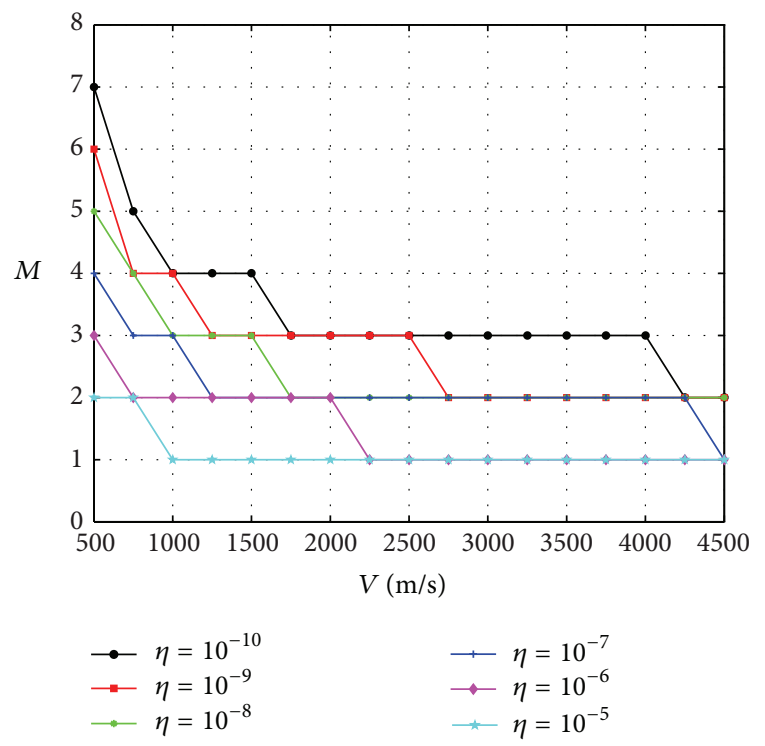

FIGURE 3: Orders of accuracy for different velocities and maximum errors. Here, $\tau=0.0001 \mathrm{~s}, h=2 \mathrm{~m}, f_{\max }=30 \mathrm{~Hz} . \eta=10^{-10}, 10^{-9}$, $10^{-8}, 10^{-7}, 10^{-6}$, and $10^{-5}$. The values of $M$ are determined by (32). When $\log _{10}|\varepsilon|<-16$, we set $\log _{10}|\varepsilon|=-16$.

\section{Modeling Results}

We adopt the high-order staggered-grid finite difference method for a horizontally layered porous model, whose parameters are shown in Table 1 . We use a $30 \mathrm{~Hz}$ Ricker wavelet in time domain and Gaussian function in space domain located at $x=400 \mathrm{~m}, z=360 \mathrm{~m}$ to generate the vibration, and the model size is $800 \mathrm{~m} \times 800 \mathrm{~m}$ (see Figure 4).

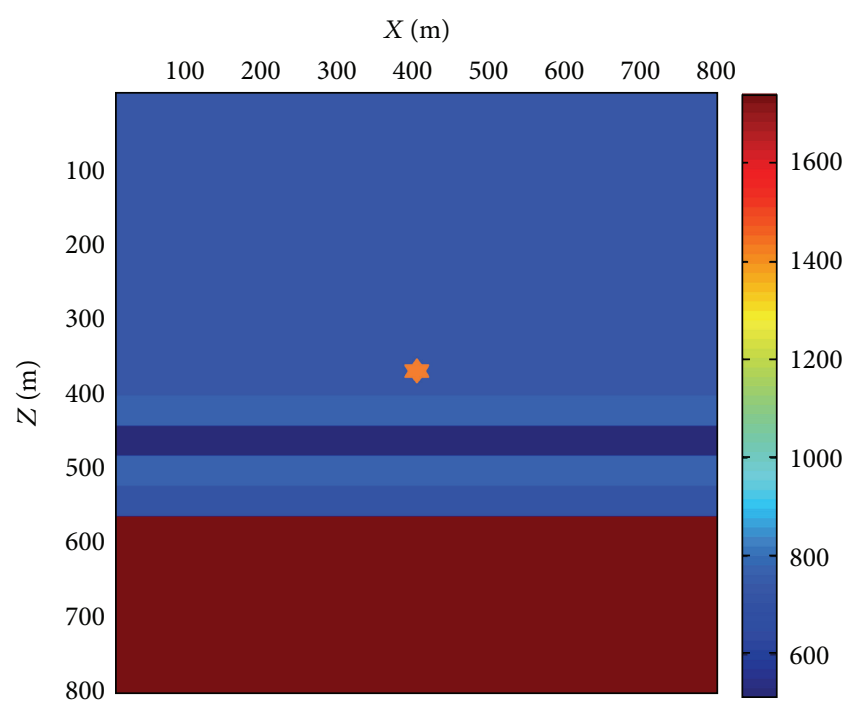

FIgURE 4: The minimum velocities for the horizontally layered porous model and the source location. A $30 \mathrm{~Hz}$ Ricker wavelet in time domain and Gaussian function in space domain located at $x=400 \mathrm{~m}, z=360 \mathrm{~m}$ is used to generate the vibration. Model size is $800 \mathrm{~m} \times 800 \mathrm{~m}$, and $h=2 \mathrm{~m}, \tau=0.0001 \mathrm{~s}$.

TABLE 2: Orders of accuracy for different velocities with the same maximum frequency.

\begin{tabular}{lcccccc}
\hline Minimum velocity $(\mathrm{m} / \mathrm{s})$ & 514 & 718 & 733 & 764 & 780 & 1740 \\
\hline$M$ & 6 & 4 & 4 & 4 & 4 & 3 \\
\hline
\end{tabular}

The orders of accuracy for this model are presented in Table 2. They are determined by (32) with $\eta=10^{-9}$, $f_{\max }=30 \mathrm{~Hz}, \tau=0.0001 \mathrm{~s}$, and $h=2 \mathrm{~m}$. Note that highorder finite difference operators provide better accuracy than low-order finite difference operators. Therefore, the highest order of accuracy used in the variable-order accuracy SFD method is adopted as the order of accuracy for the fixedorder accuracy SFD method which can be served as reference solution.

The snapshot of $z$ component of the fluid particle velocity calculated by the SFD method with variable-order accuracy (see Figure 5(b)) is almost the same as Figure 5(a), which is calculated by the SFD method with fixed-order accuracy. The same conclusion can be obtained from the comparison of Figures 5(c) and 5(d), which are the snapshots of $z$ components of the solid particle velocity calculated by the SFD method with fixed- and variable-order accuracy, respectively.

The modeling records of the $z$ components of the solid particle velocity at $x=400 \mathrm{~m}, z=400 \mathrm{~m}$ calculated by the SFD method with fixed- and variable-order accuracy are presented in Figure 6(a). The difference between them is shown in Figure 6(b). From this figure, it can be noted that the modeling results calculated by the two methods are almost identical, which demonstrate the validity of the staggeredgrid finite difference method with variable-order accuracy.

Computational efficiency of the SFD method with variable-order accuracy for this model is demonstrated by 


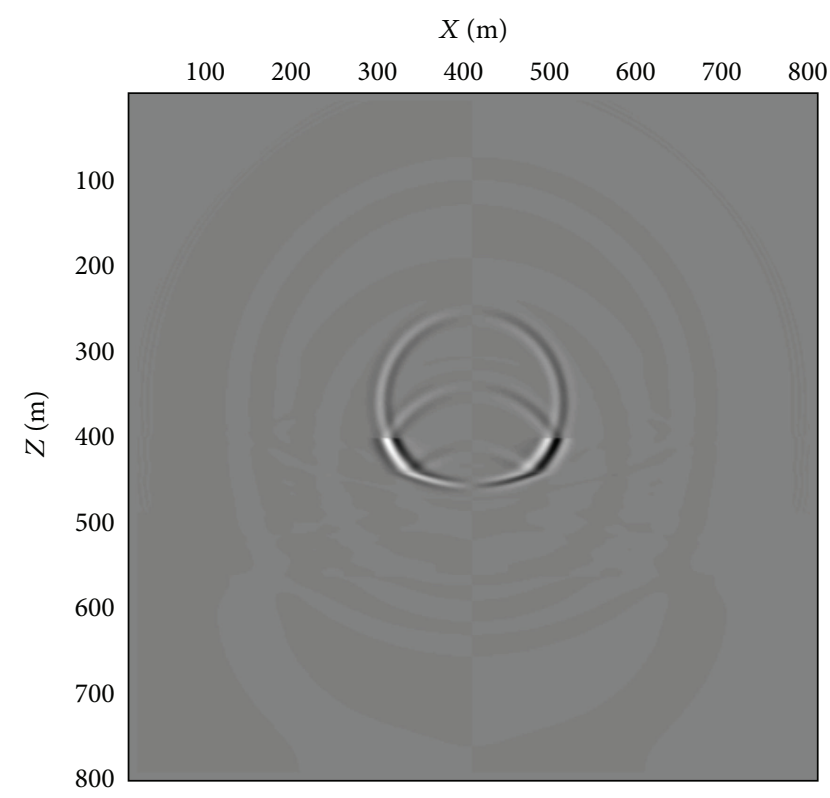

(a) The conventional method

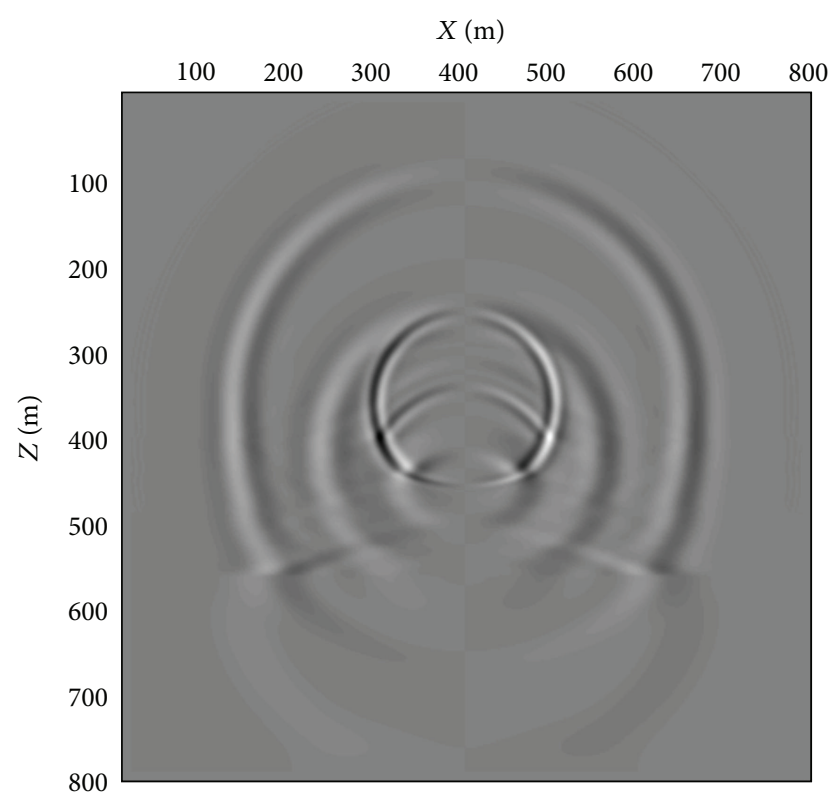

(c) The conventional method

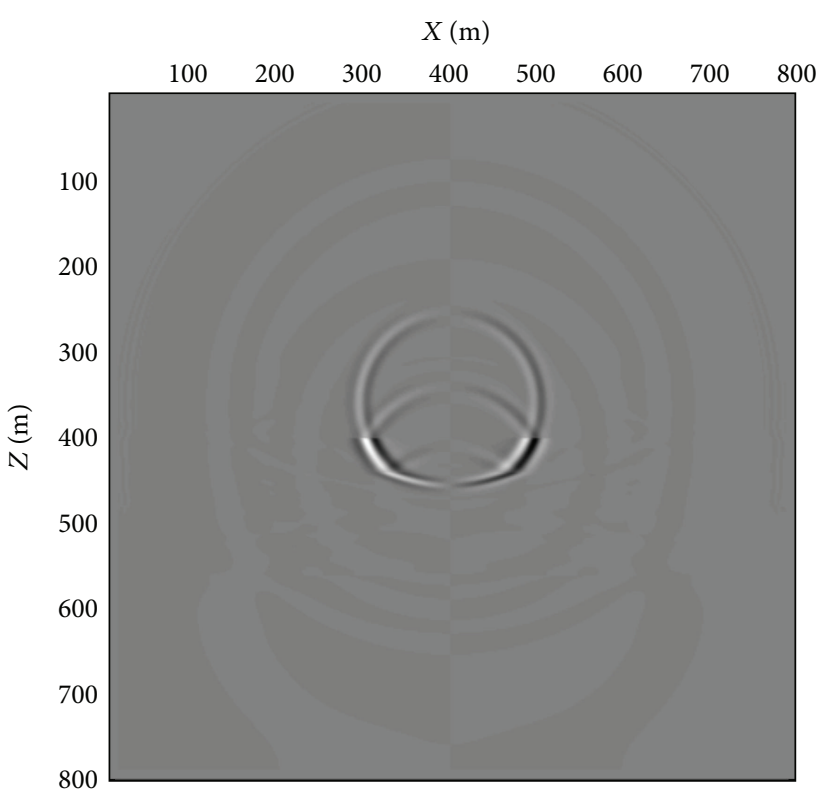

(b) The new method

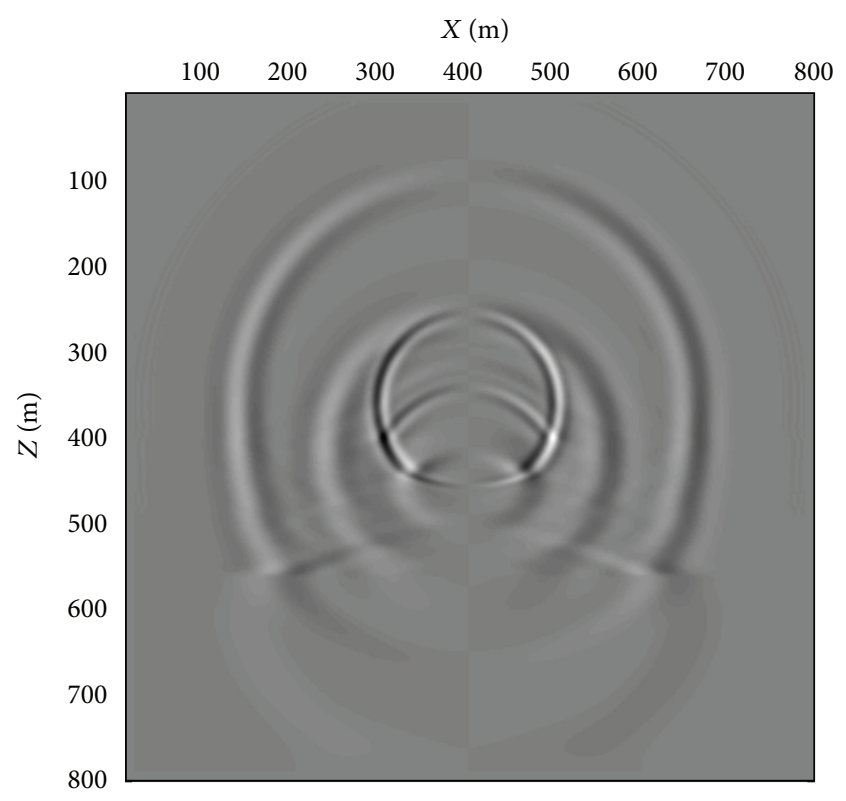

(d) The new method

Figure 5: Snapshots of the $z$ components of fluid (a)-(b) and solid (c)-(d) particle velocity at 192 ms for the horizontally layered porous model by the SFD method with fixed-order accuracy $(M=6)$ and the SFD method with variable-order accuracy $(M=6,3,3,3,3,2)$. No absorbing boundary conditions are used in the modeling.

CPU time (HP with an Intel Q8400 Core 2 quard CPU and 4.00 GB of memory), shown in Table 3. It can be seen that the SFD method with variable-order accuracy can save about $17 \%$ of the CPU time in comparison with the SFD method with fixed-order accuracy.

Data in Table 4 show the CPU time for different models. We can conclude that the efficiency depends on the characteristic of the models. That is, the savings of CPU time increase with the increase of high-velocity regions and the decrease of low-velocity regions.
The modeling results and the CPU time demonstrate that the SFD method with variable-order accuracy results in a decrease in computation time.

\section{Conclusion}

We have developed a staggered-grid finite difference scheme with variable-order accuracy for porous media. We use a method based on dispersion relation to determine the orders of accuracy for different velocity regions. The variations of 


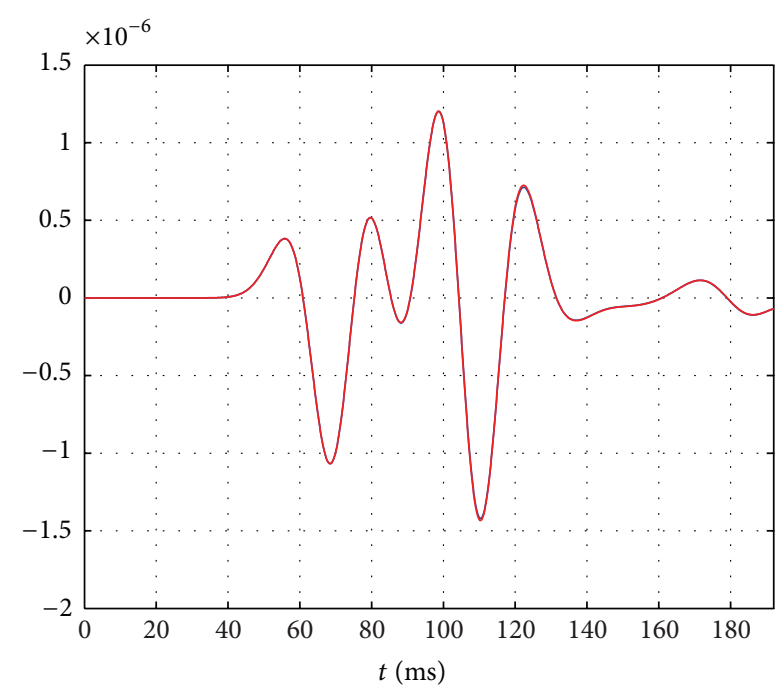

- Conventional FD method

_ Adaptive variable length spatial operator FD method

(a)

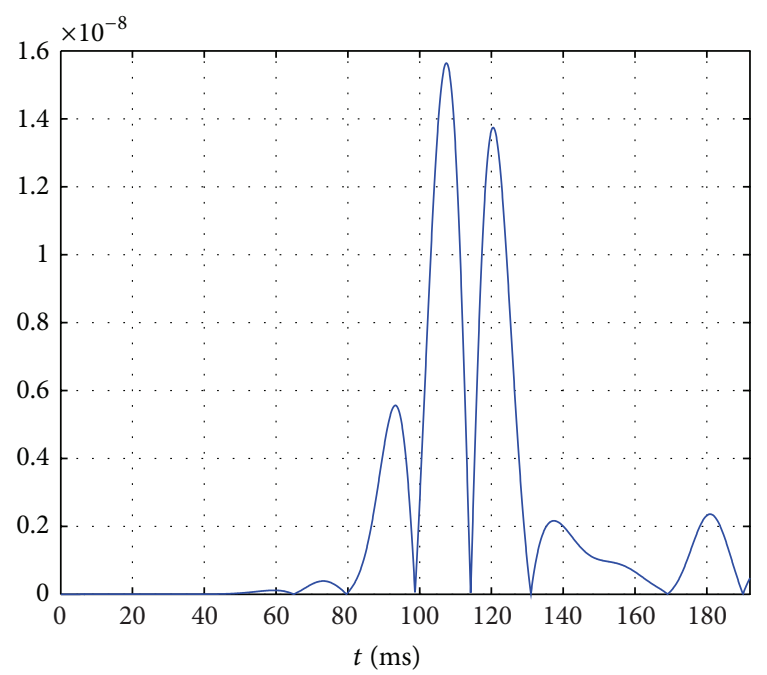

(b)

Figure 6: (a) Modeling records and (b) the difference of modeling records of the $z$ components of the solid particle velocity at $x=$ $400 \mathrm{~m}, z=400 \mathrm{~m}$ for the horizontally layered porous model by SFD schemes with fixed- and variable-order accuracy.

dispersion parameters with $k h$ or $f$ imply the validity of the new scheme. The comparison of numerical modeling results and CPU time between the SFD schemes with fixed- and variable-order accuracy indicates that the proposed scheme can enhance the computational efficiency without reducing the accuracy.

\section{Appendix}

In this appendix, we discretize the derivatives of wave equations based on high-order staggered-grid finite difference scheme for porous media.
TABLE 3: CPU time of SFD modeling for the horizontally layered model.

\begin{tabular}{lcc}
\hline $\begin{array}{l}\text { Type of SFD } \\
\text { operators }\end{array}$ & Value of $M$ & $\begin{array}{c}\text { CPU time of } 1000 \text { recursion } \\
\text { times }(\mathrm{s})\end{array}$ \\
\hline Fixed & 6 & 688 \\
Variable & $6,4,4,4,4,3$ & 572 \\
\hline
\end{tabular}

TABLE 4: CPU time of SFD modeling for different models, whose parameters are shown in Table 1 . The difference of these models is the thickness of Layer 3 and Layer 6 . Values of $M=6,4,4,4,4,3$ are used for six different velocities.

\begin{tabular}{lccc}
\hline \multicolumn{2}{c}{ Thickness (m) } & CPU time of 1000 recursion times (s) \\
Layer 3 & Layer 6 & Fixed & Variable \\
\hline 280 & 240 & 692 & 606 \\
40 & 240 & 688 & 572 \\
40 & 440 & 698 & 558 \\
\hline
\end{tabular}

The derivatives in (1) and (3) can be discretized as follows:

$$
\begin{gathered}
\frac{\partial v_{x}}{\partial t}=\frac{\left(v_{x}\right)_{p, q}^{n+1}-\left(v_{x}\right)_{p, q}^{n}}{\tau}, \\
\frac{\partial w_{x}}{\partial t}=\frac{\left(w_{x}\right)_{p, q}^{n+1}-\left(w_{x}\right)_{p, q}^{n}}{\tau}, \\
\frac{\partial \tau_{x x}}{\partial x}=\frac{1}{h} \sum_{m=1}^{M} a_{m}\left(\left(\tau_{x x}\right)_{p+(m-1 / 2), q}^{n+1 / 2}-\left(\tau_{x x}\right)_{p-(m-1 / 2), q}^{n+1 / 2}\right) \\
\frac{\partial \tau_{x z}}{\partial z}=\frac{1}{h} \sum_{m=1}^{M} a_{m}\left(\left(\tau_{x z}\right)_{p, q+(m-1 / 2)}^{n+1 / 2}-\left(\tau_{x z}\right)_{p, q-(m-1 / 2)}^{n+1 / 2}\right) \\
\frac{\partial s}{\partial x}=\frac{1}{h} \sum_{m=1}^{M} a_{m}\left((s)_{p+(m-1 / 2), q}^{n+1 / 2}-(s)_{p-(m-1 / 2), q}^{n+1 / 2}\right) .
\end{gathered}
$$

Equations (2) and (4) can be discretized using the following equations:

$$
\begin{gathered}
\frac{\partial v_{z}}{\partial t}=\frac{\left(v_{z}\right)_{p+1 / 2, q+1 / 2}^{n+1}-\left(v_{z}\right)_{p+1 / 2, q+1 / 2}^{n}}{\tau} \\
\frac{\partial w_{z}}{\partial t}=\frac{\left(w_{z}\right)_{p+1 / 2, q+1 / 2}^{n+1}-\left(w_{z}\right)_{p+1 / 2, q+1 / 2}^{n}}{\tau} \\
\frac{\partial \tau_{x z}}{\partial x}=\frac{1}{h} \sum_{m=1}^{M} a_{m}\left(\left(\tau_{x z}\right)_{p+m, q+1 / 2}^{n+1 / 2}-\left(\tau_{x z}\right)_{p-(m-1), q+1 / 2}^{n+1 / 2}\right) \\
\frac{\partial \tau_{z z}}{\partial z}=\frac{1}{h} \sum_{m=1}^{M} a_{m}\left(\left(\tau_{z z}\right)_{p+1 / 2, q+m}^{n+1 / 2}-\left(\tau_{z z}\right)_{p+1 / 2, q-(m-1)}^{n+1 / 2}\right) \\
\frac{\partial s}{\partial z}=\frac{1}{h} \sum_{m=1}^{M} a_{m}\left((s)_{p+1 / 2, q+m}^{n+1 / 2}-(s)_{p+1 / 2, q-(m-1)}^{n+1 / 2}\right)
\end{gathered}
$$


The derivatives in (7) can be discretized as follows:

$$
\begin{gathered}
\frac{\partial \tau_{x z}}{\partial t}=\frac{\left(\tau_{x z}\right)_{p, q+1 / 2}^{n+1 / 2}-\left(\tau_{x z}\right)_{p, q+1 / 2}^{n-1 / 2}}{\tau} \\
\frac{\partial v_{x}}{\partial z}=\frac{1}{h} \sum_{m=1}^{M} a_{m}\left(\left(v_{x}\right)_{p, q+m}^{n}-\left(v_{x}\right)_{p, q-(m-1)}^{n}\right) \\
\frac{\partial v_{z}}{\partial x}=\frac{1}{h} \sum_{m=1}^{M} a_{m}\left(\left(v_{z}\right)_{p+(m-1 / 2), q+1 / 2}^{n}-\left(v_{z}\right)_{p-(m-1 / 2), q+1 / 2}^{n}\right) .
\end{gathered}
$$

Equations (5), (6), and (8) can be discretized using the following equations:

$$
\begin{gathered}
\frac{\partial \tau_{x x}}{\partial t}=\frac{\left(\tau_{x x}\right)_{p+1 / 2, q}^{n+1 / 2}-\left(\tau_{x x}\right)_{p+1 / 2, q}^{n-1 / 2}}{\tau}, \\
\frac{\partial \tau_{z z}}{\partial t}=\frac{\left(\tau_{z z}\right)_{p+1 / 2, q}^{n+1 / 2}-\left(\tau_{z z}\right)_{p+1 / 2, q}^{n-1 / 2}}{\tau}, \\
\frac{\partial s}{\partial t}=\frac{(s)_{p+1 / 2, q}^{n+1 / 2}-(s)_{p+1 / 2, q}^{n-1 / 2}}{\tau}, \\
\frac{\partial v_{x}}{\partial x}=\frac{1}{h} \sum_{m=1}^{M} a_{m}\left(\left(v_{x}\right)_{p+m, q}^{n}-\left(v_{x}\right)_{p-(m-1), q}^{n}\right), \\
\frac{\partial v_{z}}{\partial z}=\frac{1}{h} \sum_{m=1}^{M} a_{m}\left(\left(w_{x}\right)_{p+m, q}^{n}-\left(w_{x}\right)_{p-(m-1), q}^{n}\left(\left(v_{z}\right)_{p+1 / 2, q+(m-1 / 2)}^{n}-\left(v_{z}\right)_{p+1 / 2, q-(m-1 / 2)}^{n}\right),\right. \\
\frac{\partial w_{z}}{\partial z}=\frac{1}{h} \sum_{m=1}^{M} a_{m}\left(\left(w_{z}\right)_{p+1 / 2, q+(m-1 / 2)}^{n}-\left(w_{z}\right)_{p+1 / 2, q-(m-1 / 2)}^{n}\right),
\end{gathered}
$$

where $h$ is the grid size, $\tau$ is the time step, $a_{m}(1,2, \ldots, M)$ are staggered-grid finite difference coefficients.

\section{Acknowledgment}

This work is supported by the National Science and Technology Major Project of China (2011ZX05023-005-009, 2011ZX05044).

\section{References}

[1] M. A. Biot, "Theory of propagation of elastic waves in a fluidsaturated porous solid. I. Low-frequency range," Acoustical Society of America, vol. 28, pp. 168-178, 1956.

[2] M. A. Biot, "Theory of propagation of elastic waves in a fluidsaturated porous solid. II. Higher frequency range," Acoustical Society of America, vol. 28, pp. 179-191, 1956.

[3] M. A. Biot, "Mechanics of deformation and acoustic propagation in porous media," vol. 33, pp. 1482-1498, 1962.
[4] J. M. Carcione, Wave Fields in Real Media: Wave Propagation in Anisotropic, Anelastic and Porous Media, Elsevier, Pergamon, 2001.

[5] J. M. Carcione, C. Morency, and J. E. Santos, "Computational poroelasticity-a review," Geophysics, vol. 75, no. 5, pp. 75A22975A243, 2010.

[6] G. Degrande and G. De Roeck, "FFT-based spectral analysis methodology for one-dimensional wave propagation in poroelastic media," Transport in Porous Media, vol. 9, no. 1-2, pp. 8597, 1992.

[7] Y. J. Masson and S. R. Pride, "Finite-difference modeling of Biot's poroelastic equations across all frequencies," Geophysics, vol. 75, no. 2, pp. N33-N41, 2010.

[8] G. Chiavassa and B. Lombard, "Time domain numerical modeling of wave propagation in 2D heterogeneous porous media," Journal of Computational Physics, vol. 230, no. 13, pp. 5288-5309, 2011.

[9] B. Dupuy, L. De Barros, S. Garambois, and J. Virieux, "Wave propagation in heterogeneous porous media formulated in the frequency-space domain using a discontinuous Galerkin method," Geophysics, vol. 76, no. 4, pp. N13-N28, 2011.

[10] G. I. Lemoine, M. Y. Ou, and R. J. LeVeque, "High-resolution finite volume modeling of wave propagation in orthotropic poroelastic media," SIAM Journal on Scientific Computing, vol. 35, no. 1, pp. B176-B206, 2013.

[11] Z. Alterman and F. Karal, "Propagation of elastic waves in layered media by finite difference methods," Bulletin of the Seismological Society of America, vol. 58, pp. 367-398, 1968.

[12] R. M. Alford, K. R. Kelly, and D. M. Boore, "Accuracy of finitedifference modeling of the acoustic wave equation," Geophysics, vol. 39, no. 6, pp. 834-841, 1974.

[13] M. Kindelan, A. Kamel, and P. Sguazzero, "On the construction and efficiency of staggered numerical differentiators for the wave equation," Geophysics, vol. 55, pp. 107-110, 1990.

[14] O. Holberg, "Computational aspects of the choice of operator and sampling interval for numerical differentiation in largescale simulation of wave phenomena," Geophysical Prospecting, vol. 35 , no. 6, pp. 629-655, 1987.

[15] R. M. Beam and R. F. Warming, "An implicit finite-difference algorithm for hyperbolic systems in conservation-law form," Journal of Computational Physics, vol. 22, no. 1, pp. 87-110, 1976.

[16] P. S. Jensen, "Finite difference techniques for variable grids," Computers and Structures, vol. 2, no. 1-2, pp. 17-29, 1972.

[17] T. Özdenvar and G. A. McMechan, "Algorithms for staggeredgrid computations for poroelastic, elastic, acoustic, and scalar wave equations," Geophysical Prospecting, vol. 45, no. 3, pp. 403420, 1997.

[18] S. Aoi and H. Fujiwara, "3D finite-difference method using discontinuous grids," Bulletin of the Seismological Society of America, vol. 89, no. 4, pp. 918-930, 1999.

[19] J. Zhang, "Quadrangle-grid velocity-stress finite difference method for poroelastic wave equations," Geophysical Journal International, vol. 139, no. 1, pp. 171-182, 1999.

[20] G. S. O'Brien, “3D rotated and standard staggered finite-difference solutions to Biot's poroelastic wave equations: stability condition and dispersion analysis," Geophysics, vol. 75, no. 4, pp. T111-T119, 2010.

[21] E. Tessmer, "Seismic finite-difference modeling with spatially varying time steps," Geophysics, vol. 65, no. 4, pp. 1290-1293, 2000. 
[22] Y. Q. Zeng, J. Q. He, and Q. H. Liu, “The application of the perfectly matched layer in numerical modeling of wave propagation in poroelastic media," Geophysics, vol. 66, no. 4, pp. 12581266, 2001.

[23] S. D. Wang, "Perfectly matched layer absorbing boundary conditions for acoustic wave equation," Oil Geophysical Prospecting, vol. 38, pp. 31-34, 2003 (Chinese).

[24] Y. Liu and M. K. Sen, "Finite-difference modeling with adaptive variable-length spatial operators," Geophysics, vol. 76, no. 4, pp. T79-T89, 2011.

[25] J. Virieux, "P-SV wave propagation in heterogeneous media: velocity-stress finite-difference method," Geophysics, vol. 51, no. 4, pp. 889-901, 1986.

[26] N. Dai, Finite difference simulation and imaging of seismic waves in complex media [Ph.D. thesis], University of Alberta, 1993.

[27] N. Dai, A. Vafidis, and E. R. Kanasewich, "Wave propagation in heterogeneous, porous media: a velocity-stress, finite-difference method," Geophysics, vol. 60, no. 2, pp. 327-340, 1995.

[28] L. G. Dong, Z. T. Ma, J. Z. Cao et al., "A staggered-grid highorder difference method of one-order elastic wave equation," Acta Geophysica Sinica, vol. 43, no. 3, pp. 411-419, 2000. 


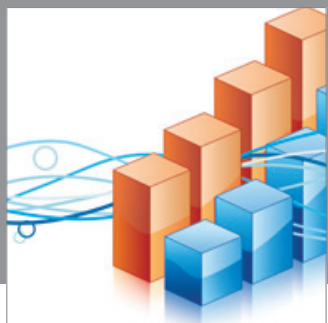

Advances in

Operations Research

mansans

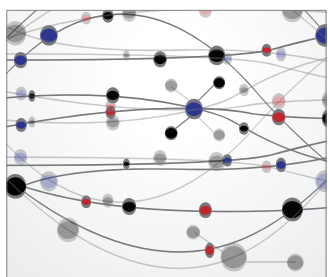

The Scientific World Journal
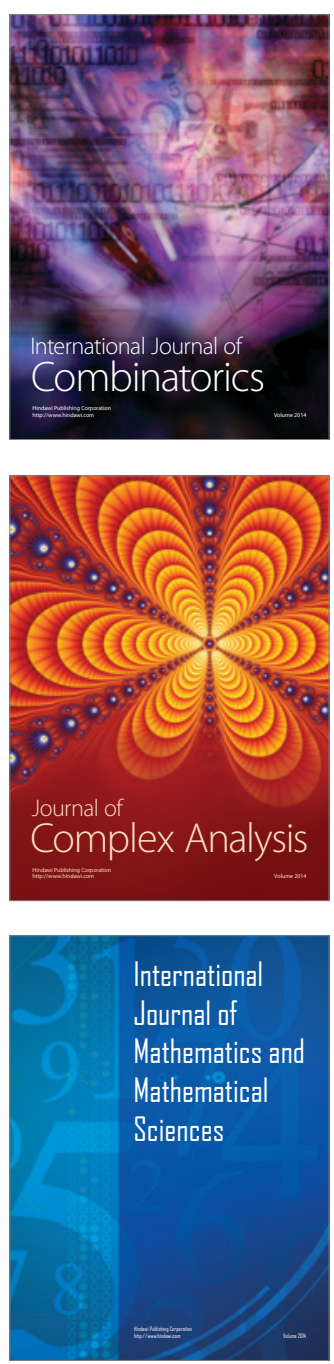
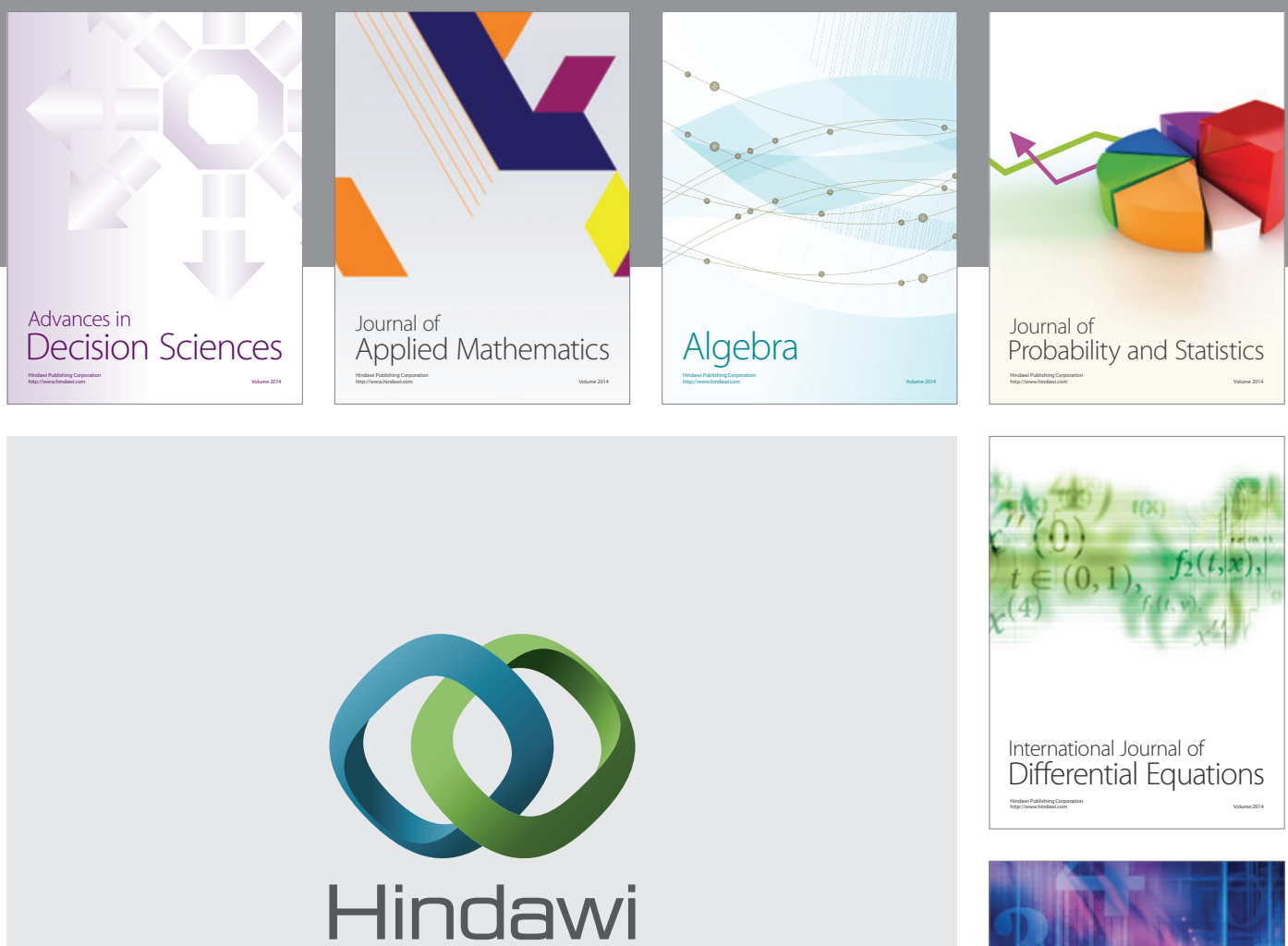

Submit your manuscripts at http://www.hindawi.com
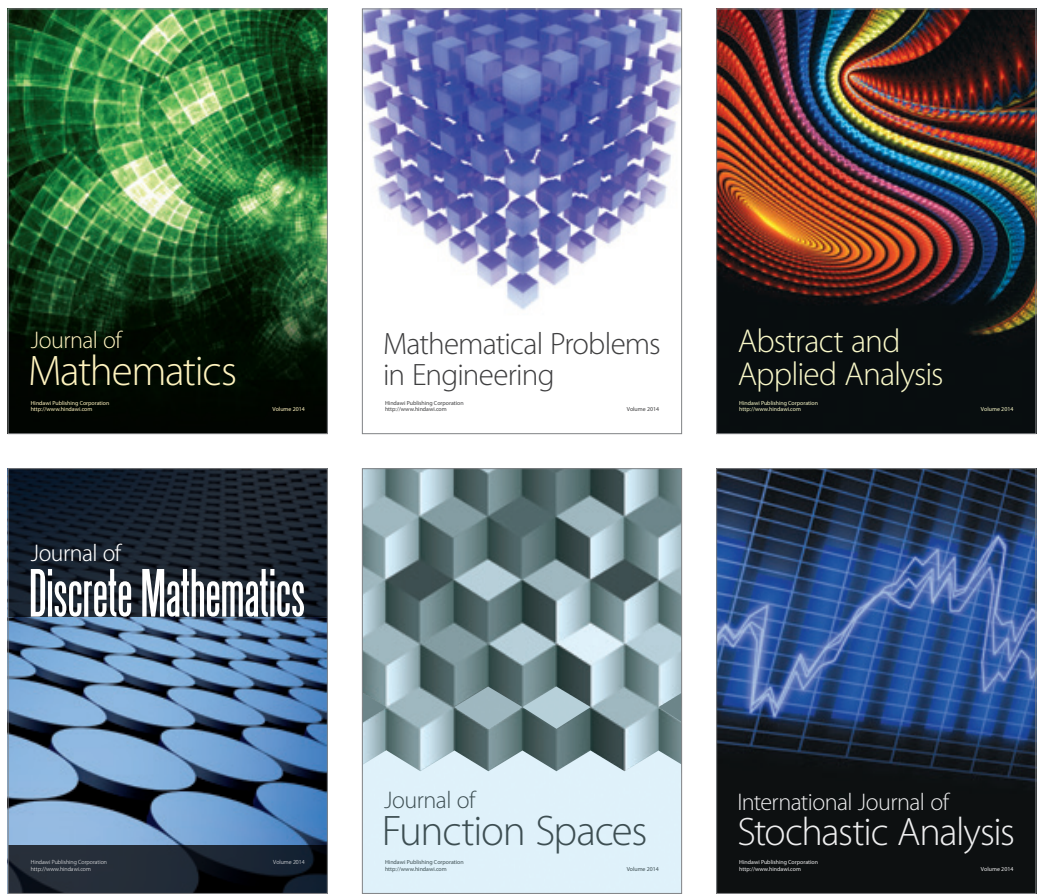

Journal of

Function Spaces

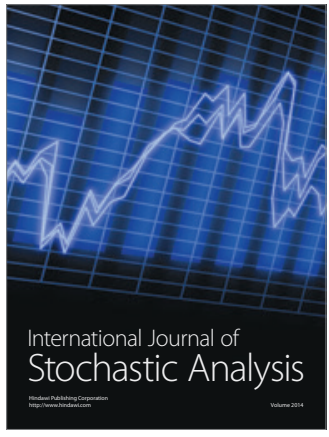

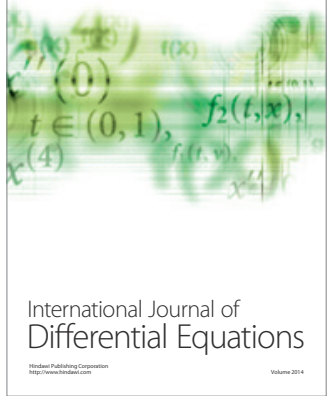
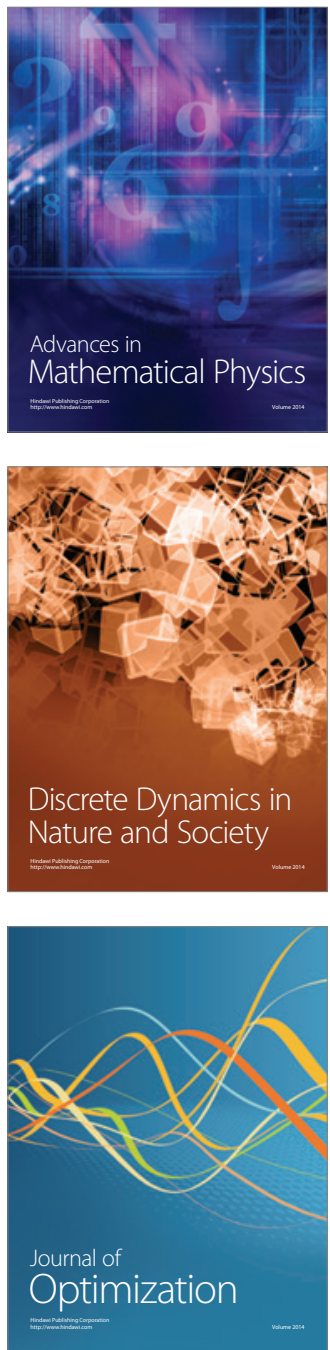